\title{
HERRAMIENTA MULTICRITERIO BASADA EN FUNCIONALIDADES SIG PARA LA IDENTIFICACIÓN DE ÁREAS POTENCIALES DE RIEGO EN ARGENTINA
}

\author{
Hidalgo García, M. (1) (P), Loyola, L (2), López, C. (3), Zunino, F. (4), Valle Vila, B. (5), San \\ Sebastián Sauto, J. (6) Hierro Rodríguez, R. (7)
}

1 Jefe de Grupo de Actuaciones y Proyectos, Gerencia de Ingeniería y Edificación (Tragsatec), Julián Camarillo 6, 28037 Madrid, mhg@tragsa.es

2 Oficial de apoyo a las inversiones en América Latina en FAO, Luis.Loyola@fao.org

${ }^{3}$ Consultora de inversiones en FAO, Celia.Lopez@fao.org

${ }^{4}$ Consultor Senior, Argentina, florzunino@gmail.com

${ }^{5}$ Responsable de actuaciones y proyectos, Gerencia de Ingeniería y Edificación (Tragsatec), Julián Camarillo 6, 28037 Madrid, bvv@tragsa.es

${ }^{6}$ Jefe de Actuaciones y Proyectos, Gerencia de Ingeniería y Edificación (Tragsatec), Julián Camarillo 6, 28037 Madrid, jsss@tragsa.es

7 Técnico Superior en Sistemas de Información Geográfica, Gerencia de SIG y Consultoría (Tragsatec), Julián Camarillo 6, 28037 Madrid, rhr@tragsa.es

\section{Resumen}

La FAO encargó a Tragsatec el diseño y desarrollo de una herramienta para la identificación de áreas potenciales de riego en Argentina en noviembre de 2013. En una primera fase se propusieron objetivos e indicadores basados en la sostenibilidad de 5 bloques temáticos (técnico-agronómico, ambiental, económico, social e institucional). Tras la recopilación de mapas, tablas y estudios enviados desde Argentina, estos datos se trataron para su uso como material capaz de establecer una priorización para la puesta en riego basado en un índice potencial de riego (IPR). Este IPR es un número entre 0 y 1 que se ha utilizado para establecer una leyenda común tanto para capas raster originales como a resultados. Una vez establecida la funcionalidad de la herramienta en un entorno Arcmap se realizaron los 5 cruces temáticos para valorar la pertinencia de los resultados. Posteriormente, se probaron combinaciones multicriterio. Se plantean una serie de conclusiones y recomendaciones sobre el uso de una herramienta informática multiparamétrica para la planificación de regadío.

\section{Abstract}

In 2014 FAO ordered Tragsatec the design and development of a decision making tool for locating potential areas to be put under irrigation in Argentina. During the first phase, there was a proposal of some goals and indicators based on the sustainability of 5 key issues (technical-agronomic, environmental, economic, social and institutional). After the collection of maps, tables and studies sent from Argentina, these data were treated to be used as a material capable of establishing a prioritization for the development of irrigation based on a potential rate of irrigation (IPR). This rate is a number between 0 and 1 which has been used to set a common legend as much for the source raster layers as for the resulting maps. Once the tool functionality in an Arcmap interface was checked, all 5 thematic layer crosses were carried on to assess the relevance of the results. Subsequently, multi-criteria combinations were tested too. To conclude, a series of conclusions and recommendations on the use of a multiparameter computer tool for irrigation planning are anticipated. 


\section{Introducción}

La FAO y el Programa de Servicios Agrícolas Provinciales de Argentina (PROSAP) cuentan con un acuerdo orientado a mejorar la eficiencia sectorial agropecuaria y las condiciones de vida de los pequeños y medianos productores mediante el incremento de las inversiones agropecuarias nacionales y regionales. Dentro de este marco, la FAO contrató en 2014 a Tragsatec para la definición de una metodología multidisciplinar para la identificación de potenciales áreas de riego complementario con el objeto de conseguir una herramienta multicriterio capaz de cruzar información gráfica de diversa índole.

Para poder dar una visión generalizada sobre el riego en Argentina se pueden ofrecer una serie de datos relevantes sobre su situación actual.

- La República Argentina cuenta con 174 Mha cultivables de las que se cultivan 39 Mha $(22,4 \%)$.

- La superficie actual regada con agua superficial abarca 1,35 Mha, con agua subterránea 0,4 Mha y con agua complementaria mixta suman 0,6 Mha. En Mendoza se riegan 13.000 ha con recursos reutilizados. El sistema de gravedad alcanza el $83 \%$, la aspersión el $12 \%$ y el localizado el $5 \%$. El $14 \%$ de la superficie es pública. La eficiencia media es de $35-40 \%$. El riego integral se da en la zona árida y el complementario en la semiárida del país.

- La superficie potencial de riego, basada en la superficie sin limitaciones y la disponibilidad de agua, se ha estimado en 16 Mha. Se prevé una ampliación a 7 Mha en los próximos 10 años.

- El sector agrícola consume $27,93 \mathrm{~km}^{3}(74 \%)$. Las aguas subterráneas suponen sólo el $30 \%$ pero son claves en regulación pluri e interanual y en sequías.

- La República cuenta con $41 \mathrm{M}$ de habitantes. Un 7\% es población rural. Población activa rural de 1,4 M hab. con sólo un $11 \%$ femenino. La fuente de abastecimiento de la población rural en zonas áridas es la subterránea.

- Los problemas de calidad en superficiales proceden de la erosión, plaguicidas, mala depuración, pozos ciegos o contaminación industrial. Existe una alta contaminación natural por As, F y Va. Las subterráneas sufren de malas prácticas y fallos de saneamiento. La red de seguimiento cuenta con 105 estaciones. Inundaciones cada vez más frecuentes. 30-40\% con problemas de salinización-sodificación.

La finalidad del trabajo era obtener resultados gráficos como sistema de apoyo a la decisión con información significativa sobre la potencialidad de regadío por zonas según la información y criterios establecidos previamente.

\section{Diseño y desarrollo de la herramienta}

Durante el periodo de diciembre de 2013 y enero de 2014 se fueron realizando diferentes envíos digitales a España para comenzar los trabajos de revisión y posterior incorporación al diseño de la herramienta. Se organizó un equipo de trabajo que contara con personal que analizara la información cartográfica y que por otro lado comenzara a proponer objetivos e indicadores viables. En Junio de 2014 se dio por finalizada la primera fase con la entrega de una versión inicial de la herramienta para la cual se habían puesto en marcha las siguientes actividades:

- Revisión de criterios de partida y objetivos del regadío en Argentina

- Definición de objetivos y consenso de los mismos para obtención de indicadores 
- Establecimiento de bloques temáticos

- Recopilación, ordenación y categorización de la información recibida

- Diagnóstico y selección de capas de uso para la herramienta

- Propuesta metodológica de la herramienta en base a un índice potencial para riego (IPR)

- Definición de las bases de funcionamiento de la herramienta multicriterio

Para el desarrollo de la herramienta se ha necesitado, por un lado revisar toda la información recibida, establecer una metodología de ponderación y asignación de valores y desarrollar un diseño y su implementación en un entorno SIG que permita manejar dicha información y la obtención de resultados cartográficos (Ver Figura 1). Para ello, se analizó toda la información de diferentes estudios temáticos realizados por las diversas empresas y la propia administración argentina en un área delimitada por 8 provincias del Nordeste Argentino.

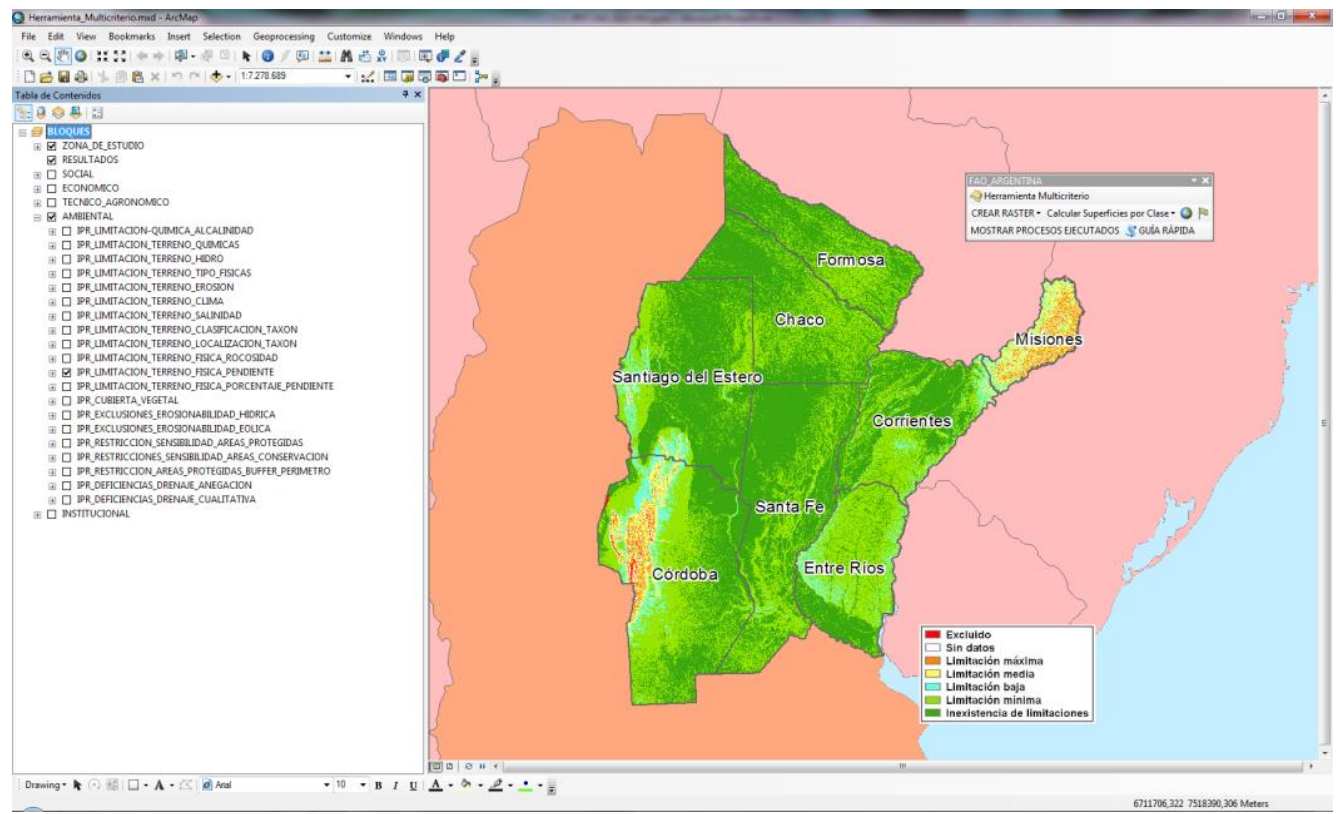

Figura 1. Aspecto general de la Herramienta Multicriterio de suma ponderada de capas raster para priorización de obras de regadío

Una vez organizada la información alfanumérica y cartográfica disponible o cuyo contenido puede someterse a una interpretación de carácter agrario sobre su potencialidad al regadío, se proponen 101 capas englobadas en 5 bloques temáticos: que puedan llegar a combinarse para evaluar la selección de áreas prioritarias para riego:

- Bloque Técnico-Agronómico

- Bloque Ambiental

- Bloque Social

- Bloque Económico

- Bloque Institucional

De esta forma y en base a la combinación de distintos factores, disponibilidad de capas cartográficas, datos alfanuméricos, limitaciones del riego, efectos potenciales y objetivos de 
la herramienta se ha calculado un denominado ÍNDICE POTENCIAL PARA RIEGO (IPR) para distintos indicadores.

Finalmente, se ha realizado una implementación personalizada de un Sistema de Información Geográfica. Se ha optado por ArcGis versión 10.1 y programación en Python con la que diseñar un interfaz que permite ponderar indicadores (capas), ponderar bloques (todos ellos en base 1 para compensar el uso de un número independiente de capas o bloques en cada combinación) y generar información de áreas potenciales de riego en formato raster con distinto tamaño de celda y cálculo de superficies.

A continuación se desglosan las diferentes tareas más relevantes llevadas a cabo para la puesta a punto de la herramienta

\subsection{Establecimiento de objetivos por bloques}

Como objetivo general del encargo de FAO se plantea el diseño de una metodología multicriterio de priorización para riego. Esta metodología ha de incorporar, por tanto, una serie de criterios compatibles con la política de FAO y el estado Argentino en cuanto a sostenibilidad general del regadío.

Se han establecido 5 bloques, coherentes con los principios de sostenibilidad de la FAO y de la República Argentina, sobre los que la priorización de zonas para su posible puesta en regadío cumpla con:

- Sostenibilidad Técnica: Apuesta por tecnologías agrarias modernas pero asumibles por la población local, adaptando el uso de los recursos y la potencialidad para riego.

- Sostenibilidad Ambiental: Estudio de condiciones ambientales actuales y futuras que garanticen la explotación del riego a medio y largo plazo sin arriesgar los recursos.

- Sostenibilidad Económica: Búsqueda de alternativas con la mayor eficiencia coste/beneficio que permitan un mantenimiento temporal a largo plazo

- Sostenibilidad Social: Fomento del desarrollo social basado en la búsqueda de la equidad y el balance de las desigualdades y una justificación social de las inversiones.

- Sostenibilidad Institucional: Análisis de la viabilidad del fomento del regadío y sometimiento de los proyectos a la legislación vigente estatal y provincial.

\subsection{Propuesta metodológica para integración de capas en la herramienta multicriterio}

Un vez establecida la información alfanumérica y cartográfica disponible sobre los 5 bloques temáticos o cuyo contenido puede someterse a una interpretación de carácter agrario sobre su potencialidad para el regadío, se propone una serie de índices que puedan llegar a combinarse para la obtención de uno o varios indicadores ambientales para la herramienta de selección de áreas prioritarias para riego.

Tabla 1. Valoración del IPR para cada indicador y leyenda estándar 


\begin{tabular}{|l|r|}
\hline \multicolumn{1}{|c|}{ CATEGORÍA } & IPR \\
\hline Exclusión de puesta en riego & NULL \\
\hline Sin dato & 0 \\
\hline Limitación máxima para riego & $0,01-0,24$ \\
\hline Limitación media para riego & $0,25-0,49$ \\
\hline Limitación baja para riego & $0,5-0,74$ \\
\hline Limitación mínima para riego & $0,75-0,99$ \\
\hline Inexistencia de limitaciones & 1 \\
\hline
\end{tabular}

De esta forma y en base a la combinación de factores, disponibilidad de capas cartográficas, datos alfanuméricos, limitaciones teóricas del riego, efectos potenciales y objetivos de la herramienta se ha calculado un denominado ÍNDICE POTENCIAL PARA RIEGO (IPR) para distintos indicadores. Como leyenda general se ha adoptado la siguiente:

Estos índices van a ser valorados numéricamente de forma que NULL indique la imposibilidad de establecer un proyecto de riego y 0,01 la máxima limitación. Desde dicho índice hasta "1" se gradúa la posibilidad de plantear limitaciones para su establecimiento según las características del parámetro de la capa correspondiente (inundabilidad del suelo, tipo de clima, presencia de población indígena, coste del bombeo a canal...).

Esta numeración debe entenderse en todo momento como un criterio de priorización según el parámetro exclusivo que se haya escogido. La categorización de los estadios intermedios es obviamente la tarea más difícil al incorporar un nivel de subjetividad o el preestablecimiento de ciertas condiciones para decidir los límites entre un valor numérico y el siguiente o el grado de separación entre ambos. Para ello es importante la valoración por distintos expertos y la retroalimentación del sistema hasta llegar a una cartografía que sea capaz de dar una interpretación válida y coherente de la priorización del riego.

Posteriormente, la combinación de diferentes índices y su ponderación relativa permitirá establecer diferentes escenarios para el regadío con mayor o menor incidencia en los aspectos multidisciplinares. Tras esta labor, la coherencia y la representatividad de los datos han de ser contrastadas con experiencias pilotos o con apoyo de expertos argentinos que homologuen las alternativas procedentes de la herramienta.

\subsection{Trabajo en diferentes niveles de detalle de la cartografía}

El nivel de detalle de los datos representados es muy variado, totalmente influenciado por la escala de la información de origen. Por este motivo, el usuario deberá tener en cuenta la escala de los datos que está combinando. Así, los bloques técnico y ambiental, contienen actualmente información mucho más detallada ya que parte de una información en origen a menor escala (local) y con divisiones naturales y los bloques social e institucional parten de una información provincial (ver Figura 2). Los económicos se reparten entre ambos tipos según la fuente utilizada en cada caso. 

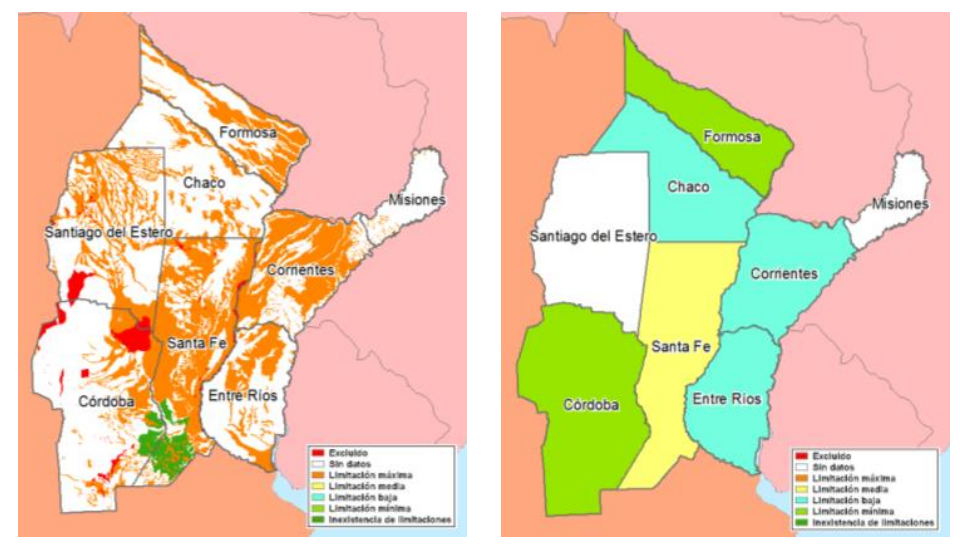

Figura 2. Comparativa entre mapa del bloque ambiental (izda.) e institucional (dcha.)

Además, en ocasiones, la cartografía de origen para indicadores iguales (suelos) parece indicar una diferencia de criterio por lo que en el resultado final podrían observarse cortes bruscos no achacables al procesado de la información sino a la información de origen.

\section{Resultados}

La herramienta SIG estaba realizada inicialmente para el programa ArcMap 10 de ESRI aunque se ha ido actualizando a las versiones 10.1 y 10.2 durante su puesta en marcha. El sistema de proyección adoptado para todas las capas es el POSGAR07. Las bandas de proyección escogidas han sido la 4 para el área de estudio y la 5 para toda Argentina. El programa requiere obligatoriamente contar con la extensión "SPATIAL ANALYST" activa para su ejecución. La escala de trabajo se puede personalizar desde una celda de $90^{*} 90$ metros hasta la que se considere, habitualmente $500 * 500$ metros.

A partir de este trabajo preliminar se ha construido una herramienta multicriterio basada en capas de 5 bloques distintos que permite seleccionar tanto el número de capas como el peso de las mismas. Para ello ha sido necesario establecer una serie de criterios de partida:

- Consta de 5 bloques diferenciados a los que habrá que asignarles un peso cuya suma debe ser igual a 1.

- Las capas raster están clasificadas en los 5 bloques ya mencionados. Se deben seleccionar las capas que vayan a participar en la operación, asignándoles individualmente el peso que tendrán dentro del bloque al que pertenecen. Nuevamente se debe tener en cuenta que la suma de tales pesos debe ser igual a 1.

- Todas las capas han sido interpretadas según criterios de priorización para riego de tal forma que el más idóneo recibía un índice 1 según el criterio para cada índice (el de menor coste, el más rentable, el mejor suelo....) ya fuera cualitativo o cuantitativo.

- En caso de exclusión el valor adjudicado es Null, equivalente a un multiplicador por cero.

- Para los elementos sin dato se adjudica el valor 0.

- Cuando se considera que dentro de ese particular indicador no existe una razón objetiva que limite la puesta en marcha del riego, se adjudica un "1".

- Todas las demás clases se ordenan según su mayor o menor limitación para el desarrollo en riego que va desde 0,99 (el más idóneo o menos limitado para regadío) a 0,01 (el menos regable o más limitado para riego). 
- Para las capas cualitativas el índice ha debido ser asignado manualmente, reinterpretando la leyenda en base a la priorización de regadío.

- Para las capas con datos numéricos se han normalizado las series como cocientes del mayor o del menor parámetro, dependiendo del criterio establecido.

- Todos los resultados de la suma ponderada, siguiendo estas bases, darán lugar a una nueva capa a la cual se le podrán aplicar los mismos criterios de partida y leyenda, pues el resultado volverá a encontrarse comprendido entre 0 y 1 ., independientemente del número de capas o bloques implicados en la consulta.

La aplicación se estructura en 5 bloques temáticos (técnico-agronómico, ambiental, social, económico y jurídico-institucional). Se utiliza una leyenda conjunta tanto para las capas individuales como para el resultado de las mismas con una simbología preestablecida, de 7 clases según el método de corte preestablecido (Figura 3).

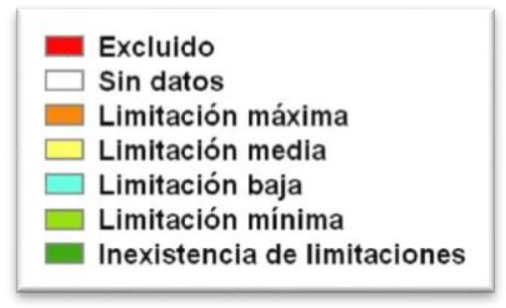

Figura 3. Leyenda única para Índice Potencial para Riego (IPR)

La herramienta incluye asociada a cada una de las capas un informe donde se documentan las fuentes, criterio de priorización y método de cálculo. Actualmente, para que la herramienta "suma ponderada" sea operativa, deben tenerse en cuenta las siguientes premisas ineludibles:

- Cuenta con un sencillo control de errores de suma de pesos. El usuario es avisado con un icono y un mensaje de atención que se deben dar los pesos de modo que sumen 1 entre bloques y entre capas dentro de cada bloque.

- Cada bloque debe tener una capa seleccionada al menos. En caso de no querer que un bloque participe en el proceso se le debe dar valor " 0 ".

- Se debe tener en cuenta que la lista de capas se ha cerrado para agilizar la gestión de datos, pero sigue siendo posible añadir capas externas. Para ello se ha habilitado un botón "+" que permitiría añadir otras capas.

- Dentro de cada bloque se deben seleccionar sólo las capas que se quieren usar en la combinación y es opcional deseleccionar el resto con el botón " $X$ " que aparece a la izquierda del listado de capas para limpiar la vista, dejando solo las participes en la suma.

- En caso de querer dejar un bloque entero sin operar se debe indicar "0" en el peso del bloque. De este modo, las capas seleccionables quedan bloqueadas para su selección.

- No se debe cambiar el nombre de cualquier dato que participe (Carpeta, geodatabase, clase de entidad, tabla, capa raster...) o la herramienta no funcionará.

De cara al manejo por parte de un usuario básico del programa se ha redactado una guía visual que sirva para la rápida inmersión en el funcionamiento. El contenido de botones, su funcionamiento y características son comentados paso a paso. 


\subsection{Escenarios temáticos}

Antes de pasar a usar la herramienta para la elaboración de mapas con escenarios complejos de combinaciones de bloques y capas con diferentes pesos se procedió a analizar la información por bloques temáticos para valorar su coherencia interna. Para ello se ha procedido a dotar a cada capa del mismo peso y operar con ellas de modo que los mapas resultantes muestren los datos según la leyenda común.

Como resultados de estos escenarios por temas (Figura 4) se marcan una serie de tendencias que sirven de referencia parcial para poder valorar la pertinencia de las capas y la viabilidad de las combinaciones.

- $\quad$ El uso de IPR permite armonizar el contenido de las tablas y su visualización. El paso previo de capas y tablas originales a capas raster con IPR requiere de un cierto tratamiento de los datos en Arcmap, pero una vez realizado permite una ponderación y una interpretación sencillas al ser independientes del número de capas o bloques utilizados en la sumatoria y el tipo de datos originales.

- La inexistencia o baja representatividad de áreas prioritarias (de limitación nula o máxima) en los mapas combinados no debe interpretarse como una ausencia de áreas preferenciales para riego sino que la suma ponderada va acumulando limitaciones parciales por lo que el análisis debe realizarse de forma comparada en el mapa resultado final como un gradiente de priorización.

- La aplicación del bloque ambiental en conjunto parece proponer una excesiva superficie excluida. Esto puede resolverse mediante una atenuación del peso del bloque en las combinaciones o una revisión de criterios, sobre todo en tablas que, usadas también como fuente en el bloque técnico, éstas se han interpretado aquí de forma más drástica.

- La colaboración de expertos de cada campo en Argentina es de gran relevancia para evaluar la relevancia de la información disponible, y valorar la adecuación de los resultados, al menos a nivel de bloque. También es interesante que se indiquen las nuevas capas de importancia contrastada de las que se carezca en la actual versión y que los especialistas puedan recomendar.

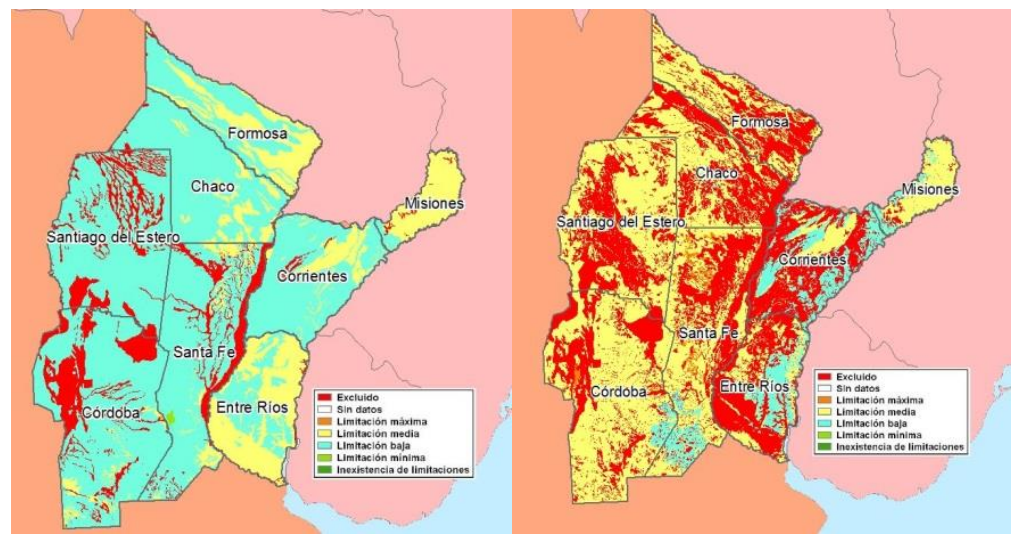




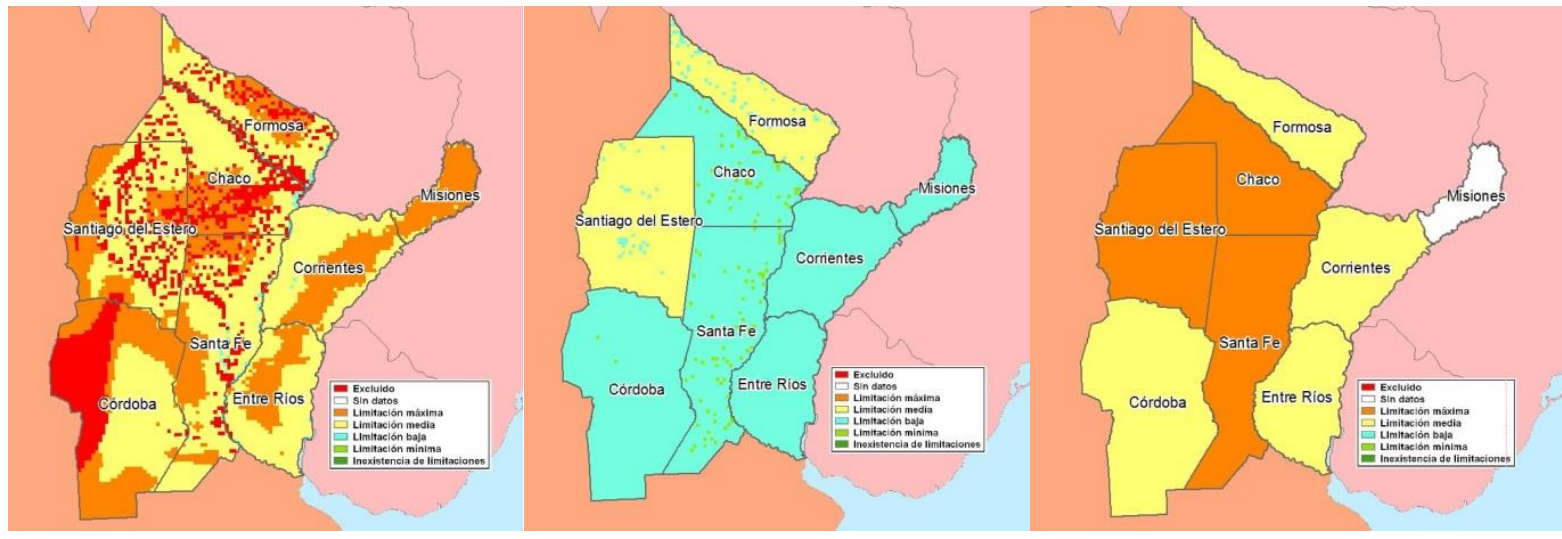

Figura 4. Combinación con mismos pesos para todas las capas del bloque (de arriba abajo y de izda a dcha): técnico, ambiental, económico, social e institucional

- Los 3 bloques con mayor detalle (Técnico, Ambiental y Económico) muestran áreas comunes: Zonas excluyentes en las Sierras de Córdoba, el valle del Salado en Santiago del Estero y Santa Fe y la ribera derecha del Paraná en Santa Fe y zonas de menor limitación situadas en las vegas Orientales de Córdoba y Santa Fe y Riberas del Río Bermejo y Río Uruguay en Corrientes y Entre Ríos.

- Los bloques social e institucional presentan datos únicamente a nivel provincial.

- Santiago del Estero y Formosa parecen inicialmente ofrecer mayores limitaciones sociales que el resto con la limitada información disponible.

- Chaco, Santiago del Estero y Santa Fe destacan institucionalmente en conjunto pero Córdoba y Buenos Aires presentan buenas capacidades para 2 de las 3 alternativas cuando se usan los pesos del estudio de partida.

- Para el bloque institucional es razonable la falta de mayor detalle por la propia naturaleza de la legislación y difícilmente ampliable por competencias no asumidas por instituciones de menor rango (departamentos o municipios) sobre un tema tan específico como la agricultura de riego.

- Para el social sería muy interesante contar con datos desglosados al menos a nivel departamental dado el papel que el riego puede y debe cumplir con el objetivo de desarrollo rural y equidad social.

\subsubsection{Escenarios globales}

Una vez contrastados los resultados obtenidos por la suma ponderada para cada uno de los 5 bloques se pasa a realizar distintas combinaciones múltiples mixtas.

ESCENARIO GLOBAL EQUITATIVO: En la primera combinación mostrada (Figura 5 izquierda) se ha realizado la suma ponderada de los 5 bloques equiparando los pesos de cada capa. Puesto que un bloque como el ambiental establecía una gran superficie excluida al tiempo que otro como el técnico permitía una baja limitación, la combinación de los 5 bloques muestra una división dicotómica entre excluida y limitación media a partes iguales. 


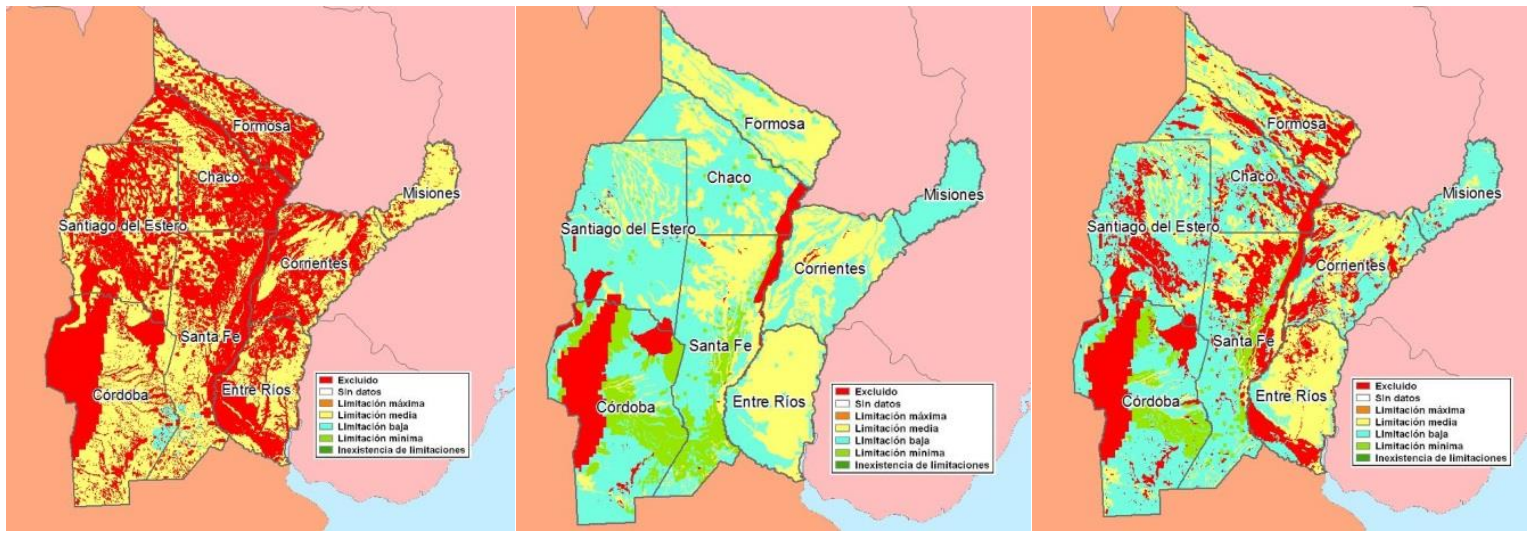

Figura 5. Combinación mixtas de 5 bloques con varias capas (de arriba abajo y de izda a dcha): Todas las capas con mismos pesos, escenario A y escenario B

ESCENARIO A: En la ponderación seleccionada en el mapa 2 (Figura 5 centro) se han escogido entre 1 y 3 capas de cada bloque. Se puede ver que la mayoría de la superficie de la zona de estudio (81\%) se enmarca entre la limitación media y baja con una exclusión y una limitación mínima entorno al 9\%. Esta combinación sería correspondiente a unos objetivos poco restrictivos donde se aplican ventajas económicas y técnicas del riego con cierta disponibilidad hídrica y pequeños inconvenientes climáticos y ambientales.

ESCENARIO B: En la siguiente cartografía (Figura 5 derecha) se ha añadido la capa de cubierta vegetal con un peso del $50 \%$ a costa de la restricción de espacios protegidos. La exclusión gana así terreno por todas las provincias. El hecho de añadir un criterio más restrictivo como la cubierta vegetal provoca que la exclusión se cuadriplique y el resto de limitaciones se vean reducidas en porcentaje. Pese a todo, aún quedarían casi 3,3 Mha dentro de la limitación mínima como áreas de priorización de proyectos de riego.

\section{Conclusiones y recomendaciones}

Tras describir las principales funciones de la herramienta en su estado de desarrollo actual se plantean unas conclusiones sobre los trabajos llevados a cabo y que sirvan para apuntar la evolución a corto plazo de la aplicación.

- La herramienta facilita una combinación de pesos y selección de capas que permite la evaluación de escenarios multicriterio para la planificación de regadío integral o complementario en la República Argentina de forma tanto gráfica como numérica.

- La herramienta actual presenta una estructura suficientemente sólida para permitir a partir de esta versión su crecimiento tanto en contenido como en posibles funciones.

- Aunque la herramienta está preparada para la incorporación de más capas y diferentes ámbitos de estudio sería recomendable nivelar más los bloques o ámbitos que no poseen un número suficiente de capas raster para poder realizar ponderaciones más equilibradas.

- La aplicación del IPR a diferentes capas interpretables es un trabajo previo a su incorporación a la herramienta, que permite, de una forma sistemática y flexible, multiplicar el número e incluso las variaciones de capas incorporables.

- EI IPR aplicado a campos numéricos resulta sencillo si se establece un criterio creciente o decreciente. En el caso de campos discretos la interpretación del IPR 
implica ciertos principios menos objetivos y que dependen del grado de conocimiento del campo en cuestión.

- En cuanto a la representación de los datos, la información de partida se presenta a distintos niveles de escala. Durante todo el proceso de valoración, se han tenido en cuenta que los datos tengan presencia en todo el ámbito de estudio, sean mensurables (base 1) y además poligonales para el cálculo de superficies.

- Se recomienda para la actualización de las capas, una escala más detallada que revierta en un incremento de la calidad y fiabilidad en la recopilación de datos, en la valoración cuantitativa y cualitativa final de los mismos.

- La significación de los resultados puede ser comprobada mediante combinaciones temáticas donde resulte más sencillo interpretar los resultados antes de que se realicen combinaciones complejas donde sea más difícil comprender los efectos de la inclusión o no de una capa o la variación de diferentes pesos relativos.

- El apoyo de técnicos especializados en distintos factores del regadío, medio ambiente, la sociedad y la economía y las instituciones es imprescindible para cotejar la correcta interpretación de las leyendas al ser convertidas en IPR y para recomendar nuevas fuentes de información.

- A la hora de establecer los escenarios resulta muy relevante establecer objetivos, puesto que los indicadores sin objetivos sólo son índices. Se recomienda la inclusión de objetivos como paso intermedio en el menú de la selección de capas dentro de cada bloque.

- Se recomienda dotar a la aplicación informática de un nombre propio, basado en un acrónimo o en un concepto de agricultura sostenible, que facilite su divulgación.

\section{Agradecimientos}

Este trabajo ha sido financiado con fondos de la FAO. 